\title{
Giant ovarian tumour: case report and literature review
}

\author{
Shazwani Adnan ${ }^{1}$, Wan Rosmidah Wan Abas², Nasuha Yaacob², Karimah Mohd², \\ Roziana Ramli ${ }^{2}$
}

${ }^{1}$ Department of Obstetrics and Gynaecology, Hospital Shah Alam, Selangor, Malaysia
${ }^{2}$ Department of Obstetrics and Gynaecology, Hospital Sultanah Nur Zahirah, Kuala Terengganu, Malaysia

Received: 18 August 2016

Accepted: 15 September 2016

*Correspondence:

Dr. Shazwani Adnan,

E-mail: shazwaniadnan@yahoo.com

Copyright: () the author(s), publisher and licensee Medip Academy. This is an open-access article distributed under the terms of the Creative Commons Attribution Non-Commercial License, which permits unrestricted non-commercial use, distribution, and reproduction in any medium, provided the original work is properly cited.

\begin{abstract}
A case of giant ovarian tumour containing total of 53 liters of serous fluid is reported here. A 61 year old postmenopausal lady presented with shortness of breath to the Casualty Department with underlying history of progressive abdominal distension for 10 years. Clinical assessment and abdominal ultrasound suggest diagnosis of giant ovarian tumour with concurrent pneumonia. Stabilization of her medical condition took priority before surgery. Both pre- and intraoperative drainage of the tumour were performed. Postoperative period was stormy but patient recovered well and was discharged on day 42 post-surgery. Histopathological examination confirmed benign papillary serous cystadenoma of the ovaries.
\end{abstract}

Keywords: Giant ovarian tumor, Serous cystadenoma, Pre and intraoperative drainage, Benign tumor

\section{INTRODUCTION}

Modern advances in medical technologies allow early detection of ovarian tumour and subsequent early surgical removal. Giant ovarian tumour is therefore a rare diagnosis. The massive size of tumour may splint the diaphragm and exert mass effect onto adjacent thoracoabdominal organs including the cardiopulmonary, gastrointestinal and major blood vessels. Removal of tumour also has considerable risks including respiratory failure, intraoperative fluid shifts, orthostatic hypotension and adynamic intestine. ${ }^{1}$ Management is therefore complicated and multidisciplinary involvement is mandatory.

\section{CASE REPORT}

A 61 year old Malay lady presented with shortness of breath to the Emergency Department. She was diagnosed with ovarian cyst and was advised for TAHBSO 10 years ago but she refused operation and defaulted follow up.
Over the years, the abdominal swelling progressively increased in size until she became bed bound 4 months prior to admission.

Clinically, the patient was thin, mildly tachypnoeic and appeared pale. She was lying down on her left side and was unable to move to a supine position or to the right side due to the massive abdominal swelling. Her BP and pulse rate were normal but she was febrile $\left(38^{\circ} \mathrm{C}\right)$. Coarse lung crepitations were heard at bilateral mid and lower zones. The abdomen was massively enlarged and tensed with prominent veins (Figure 1,2) and detection of mass was not possible clinically.

Blood investigation revealed severe iron deficiency anaemia with haemoglobin of $4.5 \mathrm{~g} / \mathrm{dL}$, elevated white cell count $17 \times 10^{3} \mathrm{~g} / \mathrm{L}$ with predominant neutrophils and hypoalbuminemia with serum albumin of $25 \mathrm{~g} / \mathrm{L}$. Serum CA 125 level was moderately elevated $(331.2 \mathrm{ng} / \mathrm{ml})$ but other tumour markers were normal. 
It was not possible to perform chest $\mathrm{x}$-ray as patient could not stand or lie flat. Transabdominal ultrasound was not helpful as the ultrasonic wave was unable to penetrate the depth of her massively distended abdomen. The patient's abdomen couldn't fit in the gantry of the CT scan machine hence evaluation by advanced imaging method was also not possible.

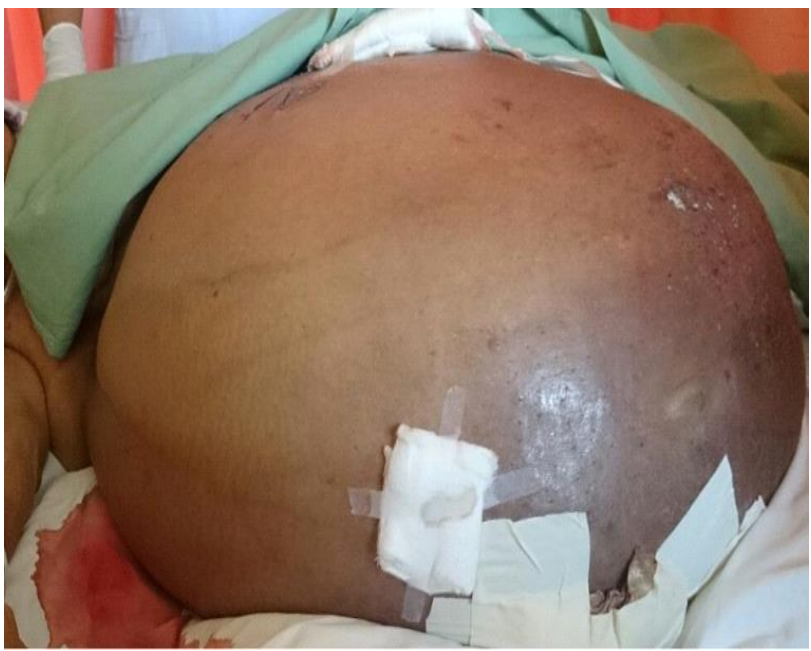

Figure 1: Massive distension of abdomen with stretched skin and prominent veins.

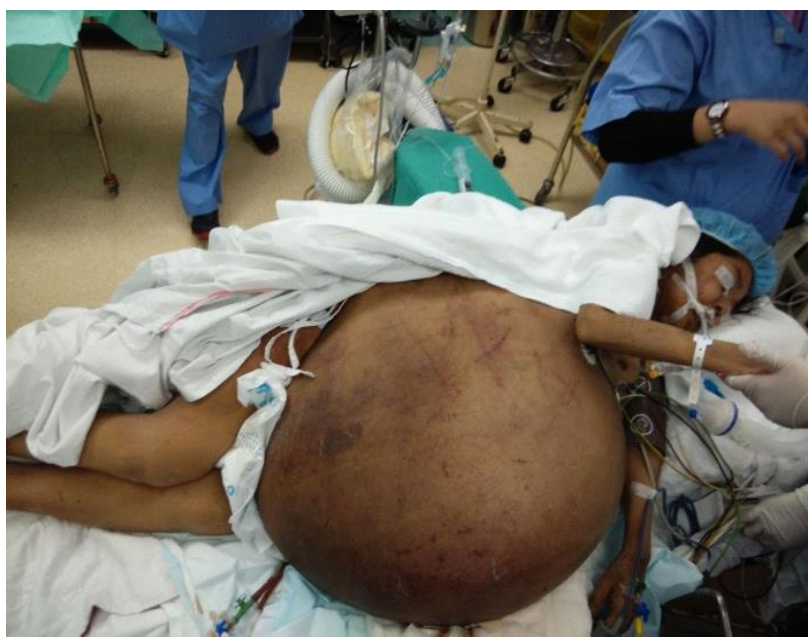

Figure 2: Patient in operating theatre intubated and prepped for surgery in left lateral position.

Differential diagnosis of giant ovarian tumour or gross ascites was made and multidisciplinary inputs from various departments were sought. She was planned for exploratory laparotomy after optimization of her medical problems and after preoperative peritoneal tapping. Total of 5 litres of straw coloured fluid was drained slowly preoperatively.

Laparotomy was performed 10 days later with the patient lying in a lateral decubitus position. A small infraumbilical midline incision (Figure 5) was made and another 48 litres of fluid was drained out from the tumour
(Figure 3) prior to surgical removal. Intraoperative findings were bilateral ovarian tumours (right cyst $30 \times 26 \times 20 \mathrm{~cm}$ weighing $4 \mathrm{~kg}$ post drainage and left cyst $15 \times 15 \mathrm{~cm}$ (Figure 4). Abdominoplasty was not performed to avoid prolonged surgery as this patient was categorized as high risk. Histopathological examination confirmed benign papillary serous cystadenoma of the ovaries.

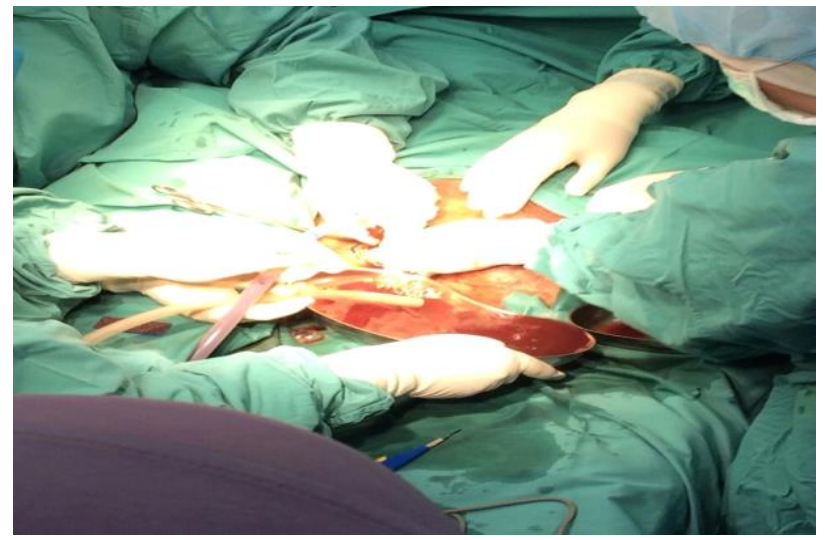

Figure 3: Drainage done prior to removal of tumour.

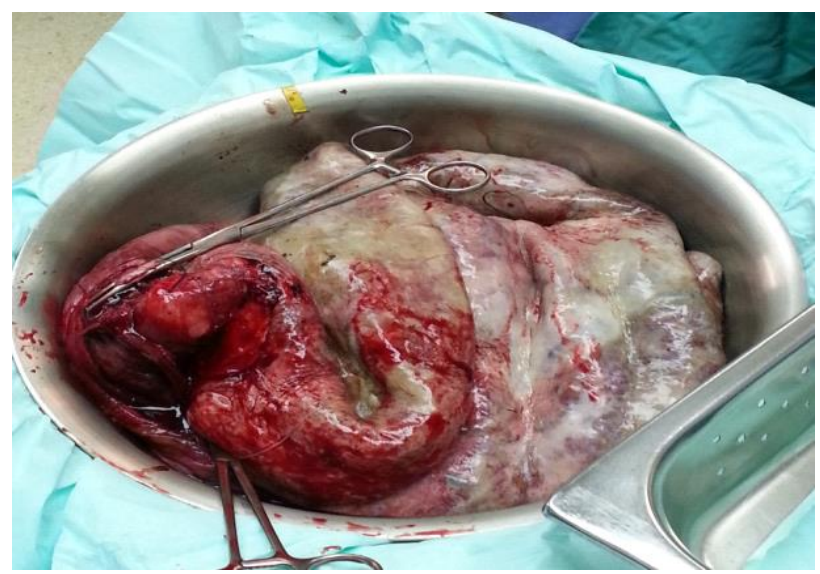

Figure 4: Giant papillary serous cystadenoma (after removal).

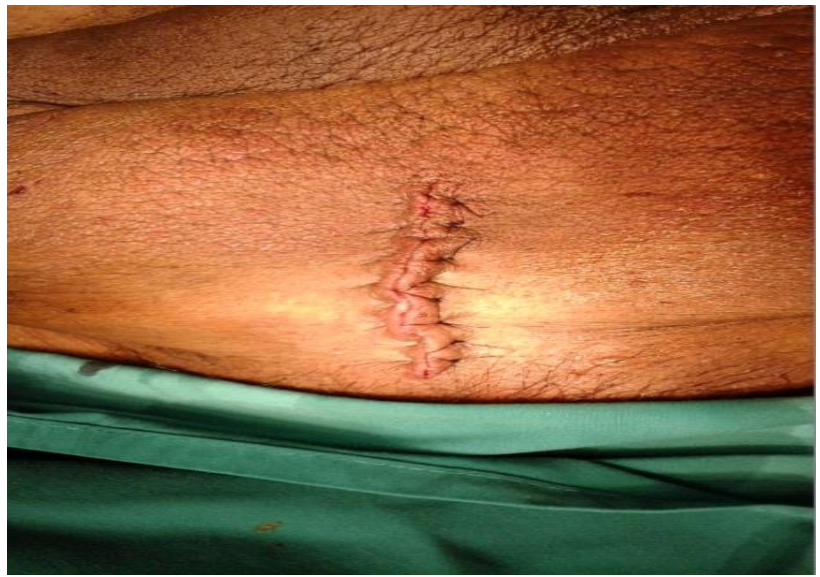

Figure 5: Low infraumbilical midline incision about $12 \mathrm{~cm}$. 
Post-operative recovery was complicated by several issues including temporary acute kidney injury secondary to massive fluid loss, septic encephalopathy and upper GIT bleeding with uraemic gastropathy. 12 days post operation the patient developed hypotensive episodes with increased abdominal drainage warranting relaparotomy. Intraoperative findings were bleeding from the abdominal wall vessels which were secured haemostatically. She required tracheostomy for prolonged intubation but later improved slowly and was discharged well after 42 days hospitalization (Figure 6).

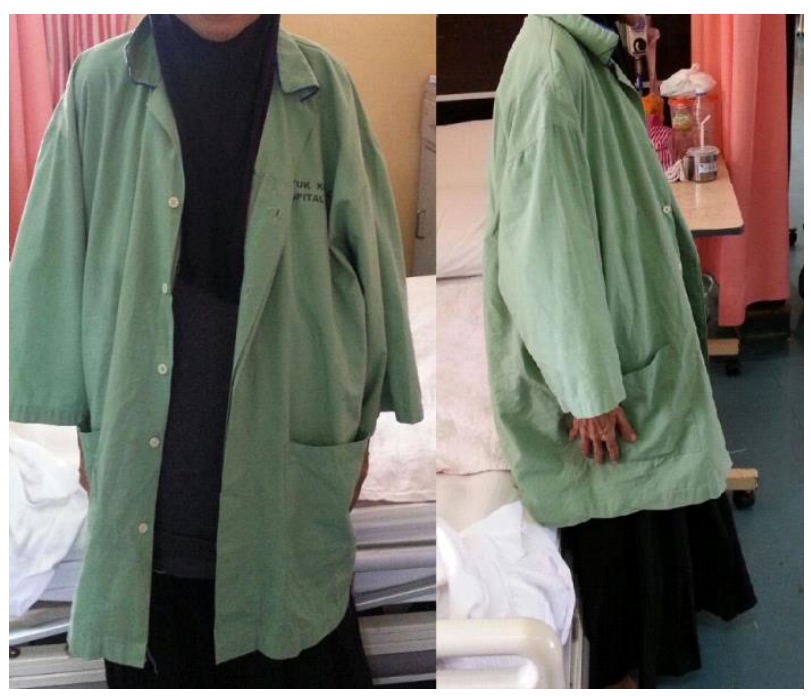

Figure 6: Patient was able to stand up and mobilize after operation, picture taken before discharge.

\section{DISCUSSION}

Giant ovarian tumour is a rare finding. The most remarkable descriptions of large ovarian enlargement are those of Spohn, ${ }^{2}$ reported one that weighed $148.6 \mathrm{~kg}$. Management of giant ovarian tumour provides significant challenges to the multidisciplinary teams before, during and even after surgery.

Preoperatively, making a diagnosis itself could be difficult. Clinical evaluation is limited when the abdomen is massively distended while imaging studies may have limited values as illustrated in this case. Cardiorespiratory compromise may occur due to splinting of diaphragm and IVC occlusion. Supine position should be avoided in case of large abdominal mass to prevent IVC compression which may lead to reduce cardiac output with sudden loss of impulse. ${ }^{3}$ IVC occlusion may also lead to troublesome and potentially fatal IVC syndrome.

Intra-operatively, cardiorespiratory issues may complicate anaesthesia especially when intra-operative fluid shift is a real possibility. ${ }^{1}$ Surgery itself may be technically difficult and in patient who is stable, input from plastic surgeon for abdominal wall cosmosis is worth considering. Apart from cosmetic indication, abdominoplasty for extremely loosened abdominal wall has been described to maintain abdominal wall competence. $^{4}$ Abdominoplasty is associated with significantly longer operating time hence not suitable in our compromised patient. Surgery itself may be associated with massive blood loss.

Prolonged postoperative ventilation failure could result from altered mechanics of ventilation as a consequence of prolonged diaphragmatic splinting. ${ }^{5}$ This is due to sudden relaxation of chronically distended flaccid abdominal and diaphragmatic musculature. ${ }^{3}$ Delayed extubation is sometimes recommended. This problem is well illustrated in this case when patient required tracheostomy for prolonged intubation. In some patients, post-operative recovery could be complicated by adynamic intestine. ${ }^{1}$ Nasogastric drainage by Ryle's tube for 72 hours prior to feeding may help to avert this complication. ${ }^{6}$

Debate continues to exist over the merits of a pre or intraoperative drainage of giant ovarian tumour. Removal of tumour in total is thought to decrease risk of spilling malignant cells. ${ }^{7}$ Einenkel $\mathrm{J}$ et al conclude that pre and intraoperative drainage should be avoided. ${ }^{8}$ From their analysis if 19 patients with giant ovarian cyst, $37 \%$ were of malignant and low malignant potential. Leakage of cyst fluid into peritoneal cavity can also cause sudden pulmonary oedema. $^{9}$

However, controlled drainage of cystic content may prevent supine hypotension syndrome. ${ }^{10}$ Intraoperative drainage of cyst fluid also prevents the phenomenon of splanchnic shock which occurs when the compressed splanchnic vessels adjacent to inferior vena cava is released with removal of mass. ${ }^{8}$ Following drainage, a small operative incision is also feasible as illustrated.

It is interesting to note that delayed bleeding from abdominal vessel occurred in our case. Madhu et al described similar complication in her patient. ${ }^{11}$ It is probable that chronic massive abdominal distension results in loss of elasticity and increase fragility of the vascular wall.

\section{CONCLUSION}

Management of giant ovarian cyst is unique and clinically challenging. Multidisciplinary inputs are necessary. Anticipation of possible complications before, during and after surgery requires careful and thoughtful planning to ensure successful outcome.

\section{Funding: No funding sources \\ Conflict of interest: None declared \\ Ethical approval: Not required}

\section{REFERENCES}

1. Pretorius RG, Matory WE Jr, La Fontaine D. Management of massive ovarian tumors. Surg Gynecol Obstet. 1989;169(6):532-6. 
2. Spohn AE. Multicystic ovarian tumour weighing 328 pounds, Texas. Med J. 1905. pp. 1273-1274.

3. Hunter DJ. Management of massive ovarian cyst. Obstet Gynecol. 1980;56(2):254-5.

4. Masamitsu K, Akira T, Yurugi S. A Case of Abdominoplasty after Removal of Giant Ovarian Cyst. Plastic and Reconstructive Surgery. 2014;2(8):e195.

5. Morrison P, Morgan G. Removal of a giant ovarian cyst. Anaesthetic and intensive care management. Anaesthesia. 1987;42:965-74.

6. Jegasothy R. Massive ovarian cysts - successful management of two cases. The Medical Journal of Malaysia. 1994;49(3):303-5.
7. Webb MJ, Decker DG, Mussey E. Factor influencing survival in stage 1 ovarian cancer. Am J Obstet Gynecol. 1973;116:222-8.

8. Einenkel J, Alexander H, Schotte D. Giant ovarian cysts: is a pre and intra operative drainage an advisable procedure. Int $\mathbf{J}$ Gynecol. Cancer. 2006;16:2039-43.

9. Drife JO, Trotter GA. Britain's biggest ovarian cyst? Br Med J. 1981;283:1661.

10. Jones DR, Vasilakis A, Pillai L, Timberlake GA. Giant, benign, mucinous cyst adenoma of the ovary: case study and literature review. Am Surg. 1992;58(7):400-3.

11. Madhu YC, Harish K. Complete resection of a giant ovarian tumour: a case report. Gynaecologic Oncology Case Reports. 2013;6:4-6.

Cite this article as: Adnan S, Wan Abas WR, Yaacob N, Mohd K, Ramli R. Giant ovarian tumour: case report and literature review. Int J Reprod Contracept Obstet Gynecol 2016;5:3601-4. 